\title{
Investigating the Effects of Selective Sampling on the Annotation Task
}

\author{
Ben Hachey, Beatrice Alex and Markus Becker \\ School of Informatics \\ University of Edinburgh \\ Edinburgh, EH8 9LW, UK \\ \{bhachey, v1balex, s0235256\}@inf.ed.ac.uk
}

\begin{abstract}
We report on an active learning experiment for named entity recognition in the astronomy domain. Active learning has been shown to reduce the amount of labelled data required to train a supervised learner by selectively sampling more informative data points for human annotation. We inspect double annotation data from the same domain and quantify potential problems concerning annotators' performance. For data selectively sampled according to different selection metrics, we find lower inter-annotator agreement and higher per token annotation times. However, overall results confirm the utility of active learning.
\end{abstract}

\section{Introduction}

Supervised training of named entity recognition (NER) systems requires large amounts of manually annotated data. However, human annotation is typically costly and time-consuming. Active learning promises to reduce this cost by requesting only those data points for human annotation which are highly informative. Example informativity can be estimated by the degree of uncertainty of a single learner as to the correct label of a data point (Cohn et al., 1995) or in terms of the disagreement of a committee of learners (Seung et al., 1992). Active learning has been successfully applied to a variety of tasks such as document classification (McCallum and Nigam, 1998), part-of-speech tagging
(Argamon-Engelson and Dagan, 1999), and parsing (Thompson et al., 1999).

We employ a committee-based method where the degree of deviation of different classifiers with respect to their analysis can tell us if an example is potentially useful. In a companion paper (Becker et al., 2005), we present active learning experiments for NER in radio-astronomical texts following this approach. ${ }^{1}$ These experiments prove the utility of selective sampling and suggest that parameters for a new domain can be optimised in another domain for which annotated data is already available.

However there are some provisos for active learning. An important point to consider is what effect informative examples have on the annotators. Are these examples more difficult? Will they affect the annotators' performance in terms of accuracy? Will they affect the annotators performance in terms of time? In this paper, we explore these questions using doubly annotated data. We find that selective sampling does have an adverse effect on annotator accuracy and efficiency.

In section 2, we present standard active learning results showing that good performance can be achieved using fewer examples than random sampling. Then, in section 3, we address the questions above, looking at the relationship between interannotator agreement and annotation time and the examples that are selected by active learning. Finally, section 4 presents conclusions and future work.

\footnotetext{
${ }^{1}$ Please refer to the companion paper for details of the selective sampling approach with experimental adaptation results as well as more information about the corpus of radioastronomical abstracts.
} 


\section{Bootstrapping NER}

The work reported here was carried out in order to assess methods of porting a statistical NER system to a new domain. We started with a NER system trained on biomedical literature and built a new system to identify four novel entities in abstracts from astronomy articles. This section introduces the Astronomy Bootstrapping Corpus (ABC) which was developed for the task, describes our active learning approach to bootstrapping, and gives a brief overview of the experiments.

\subsection{The Astronomy Bootstrapping Corpus}

The ABC corpus consists of abstracts of radio astronomical papers from the NASA Astrophysics Data System archive $^{2}$, a digital library for physics, astrophysics, and instrumentation. Abstracts were extracted from the years 1997-2003 that matched the query "quasar AND line". A set of 50 abstracts from the year 2002 were annotated as seed material and 159 abstracts from 2003 were annotated as testing material. A further 778 abstracts from the years 1997-2001 were provided as an unannotated pool for bootstrapping. On average, these abstracts contain 10 sentences with a length of 30 tokens. The annotation marks up four entity types:

Instrument-name (IN) Names of telescopes and other measurement instruments, e.g. Superconducting Tunnel Junction (STJ) camera, Plateau de Bure Interferometer, Chandra, XMM-Newton Reflection Grating Spectrometer (RGS), Hubble Space Telescope.

Source-name (SN) Names of celestial objects, e.g. NGC 7603, 3C 273, BRI 1335-0417, SDSSp J104433.04-012502.2, PC0953+ 4749.

Source-type (ST) Types of objects, e.g. Type II Supernovae (SNe II), radio-loud quasar, type 2 QSO, starburst galaxies, low-luminosity AGNs.

Spectral-feature (SF) Features that can be pointed to on a spectrum, e.g. Mg II emission, broad emission lines, radio continuum emission at 1.47 $\mathrm{GHz}, \mathrm{CO}$ ladder from (2-1) up to (7-6), non-LTE line.

\footnotetext{
${ }^{2}$ http://adsabs.harvard.edu/preprint_ service.html
}

The seed and test data sets were annotated by two astrophysics PhD students. In addition, they annotated 1000 randomly sampled sentences from the pool to provide a random baseline for active learning. These sentences were doubly annotated and adjudicated and form the basis for our calculations in section 3.

\subsection{Inter-Annotator Agreement}

In order to ensure consistency in annotation projects, corpora are often annotated by more than one annotator, e.g. in the annotation of the Penn Treebank (Marcus et al., 1994). In these cases, inter-annotator agreement is frequently reported between different annotated versions of a corpus as an indicator for the difficulty of the annotation task. For example, Brants (2000) reports inter-annotator agreement in terms of accuracy and f-score for the annotation of the German NEGRA treebank.

Evaluation metrics for named entity recognition are standardly reported as accuracy on the token level, and as f-score on the phrasal level, e.g. Sang (2002), where token level annotation refers to the B-I-O coding scheme. ${ }^{3}$ Likewise, we will use accuracy to report inter-annotator agreement on the token level, and f-score for the phrase level. We may arbitrarily assign one annotator's data as the gold standard, since both accuracy and f-score are symmetric with respect to the test and gold set. To see why this is the case, note that accuracy can simply be defined as the ratio of the number of tokens on which the annotators agree over the total number of tokens. Also the f-score is symmetric, since recall $(\mathrm{A}, \mathrm{B})=\operatorname{precision}(\mathrm{B}, \mathrm{A})$ and (balanced) $\mathrm{f}$-score is the harmonic mean of recall and precision (Brants, 2000). The pairwise f-score for the ABC corpus is 85.52 (accuracy of 97.15) with class information and 86.15 (accuracy of 97.28) without class information. The results in later sections will be reported using this pairwise f-score for measuring agreement.

For NER, it is also common to compare an annotator's tagged document to the final, reconciled version of the document, e.g. Robinson et al. (1999) and Strassel et al. (2003). The inter-annotator fscore agreement calculated this way for MUC-7 and Hub 4 was measured at 97 and 98 respectively. The

\footnotetext{
${ }^{3} \mathrm{~B}-\mathrm{X}$ marks the beginning of a phrase of type $\mathrm{X}$, I-X denotes the continuation of an $\mathrm{X}$ phrase, and $\mathrm{O}$ a non-phrasal token.
} 
doubly annotated data for the ABC corpus was resolved by the original annotators in the presence of an astronomy adjudicator (senior academic staff) and a computational linguist. This approach gives an f-score of 91.89 (accuracy of 98.43) with class information for the ABC corpus. Without class information, we get an f-score of 92.22 (accuracy of 98.49), indicating that most of our errors are due to boundary problems. These numbers suggest that our task is more difficult than the generic NER tasks from the MUC and HUB evaluations.

Another common agreement metric is the kappa coefficient which normalises token level accuracy by chance, e.g. Carletta et al. (1997). This metric showed that the human annotators distinguish the four categories with a reproducibility of $\mathrm{K}=.925$ $(\mathrm{N}=44775, \mathrm{k}=2$; where $\mathrm{K}$ is the kappa coefficient, $\mathrm{N}$ is the number of tokens and $\mathrm{k}$ is the number of annotators).

\subsection{Active Learning}

We have already mentioned that there are two main approaches in the literature to assessing the informativity of an example: the degree of uncertainty of a single learner and the disagreement between a committee of learners. For the current work, we employ query-by-committee (QBC). We use a conditional Markov model (CMM) tagger (Klein et al., 2003; Finkel et al., 2005) to train two different models on the same data by splitting the feature set. In this section we discuss several parameters of this approach for the current task.

Level of annotation For the manual annotation of named entity examples, we needed to decide on the level of granularity. The question arises of what constitutes an example that will be submitted to the annotators. Possible levels include the document level, the sentence level and the token level. The most finegrained annotation would certainly be on the token level. However, it seems unnatural for the annotator to label individual tokens. Furthermore, our machine learning tool models sequences at the sentence level and does not allow to mix unannotated tokens with annotated ones. At the other extreme, one may submit an entire document for annotation. A possible disadvantage is that a document with some interesting parts may well contain large portions with re- dundant, already known structures for which knowing the manual annotation may not be very useful. In the given setting, we decided that the best granularity is the sentence.

Sample Selection Metric There are a variety of metrics that could be used to quantify the degree of deviation between classifiers in a committee (e.g. KL-divergence, information radius, f-measure). The work reported here uses two sentence-level metrics based on KL-divergence and one based on $\mathrm{f}$ measure.

$K L$-divergence has been used for active learning to quantify the disagreement of classifiers over the probability distribution of output labels (McCallum and Nigam, 1998; Jones et al., 2003). It measures the divergence between two probability distributions $p$ and $q$ over the same event space $\chi$ :

$$
D(p \| q)=\sum_{x \in \chi} p(x) \log \frac{p(x)}{q(x)}
$$

KL-divergence is a non-negative metric. It is zero for identical distributions; the more different the two distributions, the higher the KL-divergence. Intuitively, a high KL-divergence score indicates an informative data point. However, in the current formulation, KL-divergence only relates to individual tokens. In order to turn this into a sentence score, we need to combine the individual KL-divergences for the tokens within a sentence into one single score. We employed mean and max.

The $f$-complement has been suggested for active learning in the context of NP chunking as a structural comparison between the different analyses of a committee (Ngai and Yarowsky, 2000). It is the pairwise f-measure comparison between the multiple analyses for a given sentence:

$$
f_{\text {comp }}^{\mathcal{M}}=\frac{1}{2} \sum_{M, M^{\prime} \in \mathcal{M}}\left(1-F_{1}\left(M(t), M^{\prime}(t)\right)\right)
$$

where $F_{1}$ is the balanced f-measure of $M(t)$ and $M^{\prime}(t)$, the preferred analyses of data point $t$ according to different members $M, M^{\prime}$ of ensemble $\mathcal{M}$. We take the complement so that it is oriented the same as KL-divergence with high values indicating high disagreement. This is equivalent to taking the inter-annotator agreement between $|\mathcal{M}|$ classifiers. 


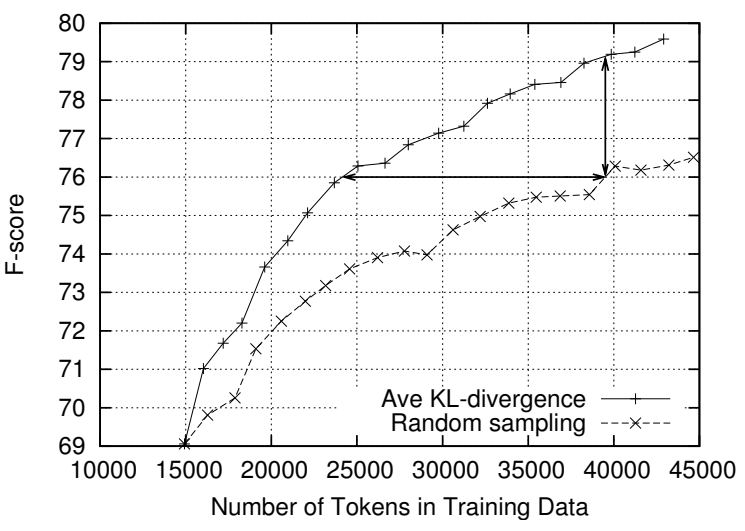

Figure 1: Learning curve of the real AL experiment.

\subsection{Experiments}

To tune the active learning parameters discussed in section 2.3, we ran detailed simulated experiments on the named entity data from the BioNLP shared task of the COLING 2004 International Joint Workshop on Natural Language Processing in Biomedicine and its Applications (Kim et al., 2004). These results are treated in detail in the companion paper (Becker et al., 2005).

We used the CMM tagger to train two different models by splitting the feature set to give multiple views of the same data. The feature set was handcrafted such that it comprises different views while empirically ensuring that performance is sufficiently similar. On the basis of the findings of the simulation experiments we set up the real active learning annotation experiment using: average KL-divergence as the selection metric and a feature split that divides the full feature set roughly into features of words and features derived from external resources. As smaller batch sizes require more retraining iterations and larger batch sizes increase the amount of annotation necessary at each round and could lead to unnecessary strain for the annotators, we settled on a batch size of 50 sentences for the real AL experiment as a compromise between computational cost and work load for the annotator.

We developed an active annotation tool and ran real annotation experiments on the astronomy abstracts described in section 2.1. The tool was given to the same astronomy $\mathrm{PhD}$ students for annotation who were responsible for the seed and test data. The learning curve for selective sampling is plotted in figure 1.4 The randomly sampled data was doubly annotated and the learning curve is averaged between the two annotators.

Comparing the selective sampling performance to the baseline, we confirm that active learning provides a significant reduction in the number of examples that need annotating. In fact, the random curve reaches an f-score of 76 after approximately 39000 tokens have been annotated while the selective sampling curve reaches this level of performance after only $\approx 24000$ tokens. This represents a substantial reduction in tokens annotated of $38.5 \%$. In addition, at 39000 tokens, selectively sampling offers an error reduction of $21.4 \%$ with a 3 point improvement in f-score.

\section{Evaluating Selective Sampling}

Standardly, the evaluation of active learning methods and the comparison of sample selection metrics draws on experiments over gold-standard annotated corpora, where a set of annotated data is at our disposal, e.g. McCallum and Nigam (1998), Osborne and Baldridge (2004). This assumes implicitly that annotators will always produce gold-standard quality annotations, which is typically not the case, as we discussed in Section 2.2. What is more, we speculate that annotators might have an even higher error rate on the supposedly more informative, but possibly also more difficult examples. However, this would not be reflected in the carefully annotated and verified examples of a gold standard corpus. In the following analysis, we leverage information from doubly annotated data to explore the effects on annotation of selectively sampled examples.

To evaluate the practicality and usefulness of active learning as a generally applicable methodology, it is desirable to be able to observe the behaviour of the annotators. In this section, we will report on the evaluation of various subsets of the doubly annotated portion of the $\mathrm{ABC}$ corpus comprising 1000 sentences, which we sample according to a sample selection metric. That is, examples are added to the subsets according to the sample selection metric, selecting those with higher disagreement first. This allows us to trace changes in inter-annotator agree-

\footnotetext{
${ }^{4}$ Learning curves reflect the performance on the test set using the full feature set.
} 
ment between the full corpus and selected subsets thereof. Also, we will inspect timing information. This novel methodology allows us to experiment with different sample selection metrics without having to repeat the actual time and resource intensive annotation.

\subsection{Error Analysis}

To investigate the types of classification errors, it is common to set up a confusion matrix. One approach is to do this at the token level. However, we are dealing with phrases and our analysis should reflect that. Thus we devised a method for constructing a confusion matrix based on phrasal alignment. These confusion matrices are constructed by giving a double count for each phrase that has matching boundaries and a single count for each phrase that does not have matching boundaries. To illustrate, consider the following sentences-annotated with phrases A, B, and C for annotator 1 on top and annotator 2 on bottomas sentence 1 and sentence 2 respectively:

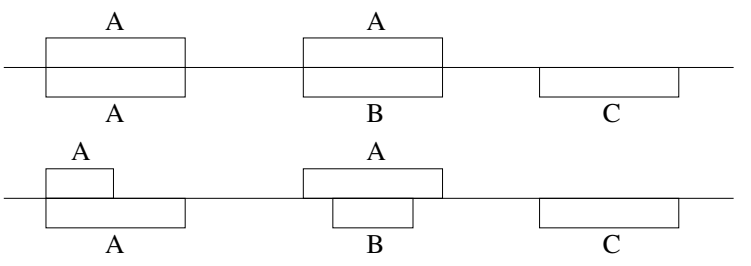

Sentence 1 will get a count of 2 for $\mathrm{A} / \mathrm{A}$ and for $\mathrm{A} / \mathrm{B}$ and a count of 1 for $\mathrm{O} / \mathrm{C}$, while sentence 2 will get 2 counts of $\mathrm{A} / \mathrm{O}$, and 1 count each of $\mathrm{O} / \mathrm{A}$, $\mathrm{O} / \mathrm{B}$, and $\mathrm{O} / \mathrm{C}$. Table 1 contains confusion matrices for the first 100 sentences sorted by averaged KLdivergence and for the full set of 1000 random sentences from the pool data. (Note that these confusion matrices contain percentages instead of raw counts so they can be directly compared.)

We can make some interesting observations looking at these phrasal confusion matrices. The main effect we observed is the same as was suggested by the f-score inter-annotator agreement errors in section 2.1. Specifically, looking at the full random set of 1000 sentences, almost all errors (Where $*$ is any entity phrase type, $\frac{* / O+O / * \text { errors }}{\text { all errors }}=95.43 \%$ ) are due to problems with phrase boundaries. Comparing the full random set to the 100 sentences with the highest averaged KL-divergence, we can see that this is even more the case for the sub-set of 100 sentences $(97.43 \%)$. Therefore, we can observe that
100: A2

A1

\begin{tabular}{c|c|c|c|c|c|}
\multicolumn{2}{c}{} & \multicolumn{3}{c}{ A2 } \\
\multicolumn{1}{c}{} & \multicolumn{1}{c}{ IN } & \multicolumn{1}{c}{ SN } & \multicolumn{1}{c}{ ST } & \multicolumn{1}{c}{ SF } & \multicolumn{1}{c}{ O } \\
\cline { 2 - 6 } IN & 12.0 & 0.0 & 0.0 & 0.0 & 0.4 \\
\cline { 2 - 6 } SN & 0.0 & 10.4 & 0.0 & 0.0 & 0.4 \\
\cline { 2 - 6 } ST & 0.0 & 0.4 & 30.3 & 0.0 & 1.0 \\
\cline { 2 - 6 } SF & 0.0 & 0.0 & 0.0 & 31.1 & 3.9 \\
\cline { 2 - 6 } O & 0.2 & 0.4 & 2.9 & 6.4 & - \\
\cline { 2 - 6 } & & & & &
\end{tabular}

1000:

A2

\begin{tabular}{|c|c|c|c|c|c|}
\hline & IN & SN & ST & SF & 0 \\
\hline IN & 9.4 & 0.0 & 0.0 & 0.0 & 0.3 \\
\hline SN & 0.0 & 10.1 & 0.2 & 0.1 & 0.3 \\
\hline ST & 0.0 & 0.1 & 41.9 & 0.1 & 1.6 \\
\hline SF & 0.0 & 0.0 & 0.1 & 25.1 & 3.0 \\
\hline $\mathrm{O}$ & 0.3 & 0.2 & 2.4 & 4.8 & - \\
\hline
\end{tabular}

Table 1: Phrasal confusion matrices for document sub-set of 100 sentences sorted by average KLdivergence and for full random document sub-set of 1000 sentences (A1: Annotator 1, A2: Annotator 2).

\begin{tabular}{|l|c|c|}
\hline Entity & 100 & 1000 \\
\hline \hline Instrument-name & $12.4 \%$ & $9.7 \%$ \\
Source-name & $10.8 \%$ & $10.7 \%$ \\
Source-type & $31.7 \%$ & $43.7 \%$ \\
Spectral-feature & $35.0 \%$ & $28.2 \%$ \\
O & $9.9 \%$ & $7.7 \%$ \\
\hline
\end{tabular}

Table 2: Normalised distributions of agreed entity annotations.

there is a tendency for the averaged KL-divergence selection metric to choose sentences where phrase boundary identification is difficult.

Furthermore, comparing the confusion matrices for 100 sentences and for the full set of 1000 shows that sentences containing less common entity types tend to be selected first while sentences containing the most common entity types are dispreferred. Table 2 contains the marginal distribution for annotator 1 (A1) from the confusion matrices for the ordered sub-set of 100 and for the full random set of 1000 sentences. So, for example, the sorted sub-set contains $12.4 \%$ Instrument-name annotations (the least common entity type) while the full set contains $9.7 \%$. And, $31.7 \%$ of agreed entity annotations in the first sub-set of 100 are Source-type (the most common entity type), whereas the propor- 


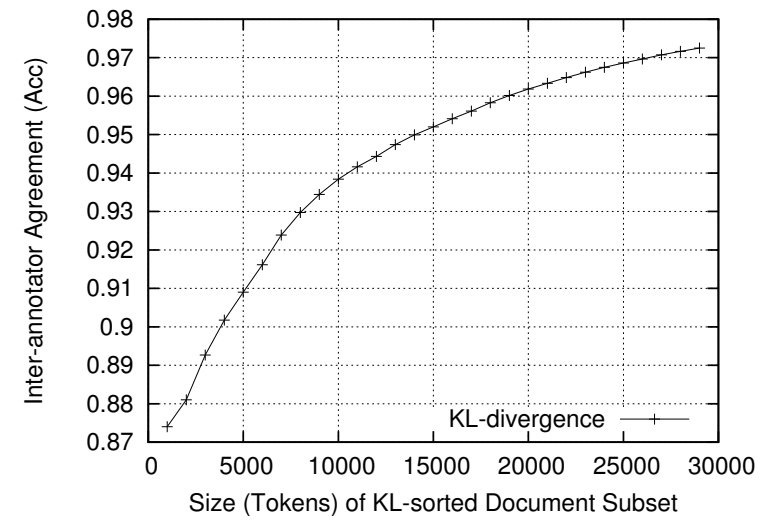

Figure 2: Raw agreement plotted against KL-sorted document subsets.

tion of agreed Source-type annotations in the full random set is $43.7 \%$. Looking at the $\mathrm{O}$ row, we also observe that sentences with difficult phrases are preferred. A similar effect can be observed in the marginals for annotator 2 .

\subsection{Annotator Performance}

So far, the behaviour we have observed is what you would expect from selective sampling; there is a marked improvement in terms of cost and error rate reduction over random sampling. However, selective sampling raises questions of cognitive load and the quality of annotation. In the following section we investigate the relationship between informativity, inter-annotator agreement, and annotation time.

While reusability of selective samples for other learning algorithms has been explored (Baldridge and Osborne, 2004), no effort has been made to quantify the effect of selective sampling on annotator performance. We concentrate first on the question: Are informative examples more difficult to annotate? One way to quantify this effect is to look at the correlation between human agreement and the token-level KL-divergence. The Pearson correlation coefficient indicates the degree to which two variables are related. It ranges between -1 and 1 , where 1 means perfectly positive correlation, and -1 perfectly negative correlation. A value of 0 indicates no correlation. The Pearson correlation coefficient on all tokens gives a very weak correlation coefficient of $-0.009 .^{5}$ However, this includes many trivial to-

\footnotetext{
${ }^{5}$ In order to make this calculation, we give token-level agree-
}

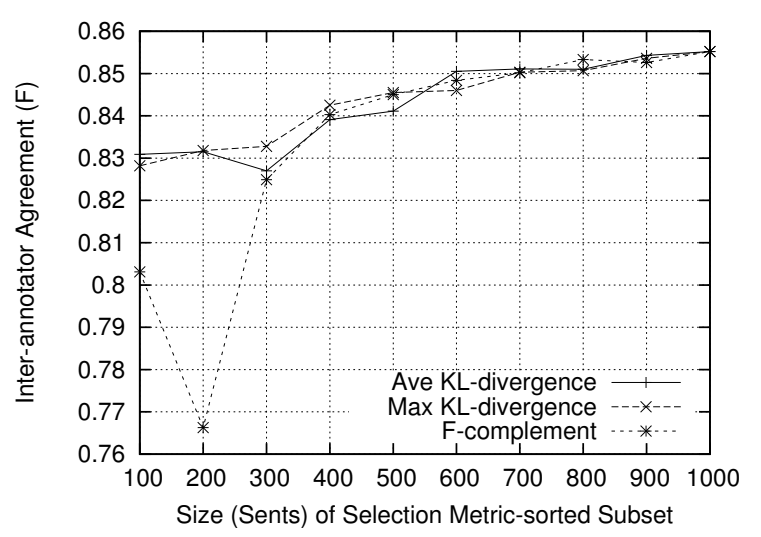

Figure 3: Human disagreement plotted against selection metric-sorted document subsets.

kens which are easily identified as being outside an entity phrase. If we look just at tokens that at least one of the annotators posits as being part of an entity phrase, we observe a larger effect with a Pearson correlation coefficient of -0.120 , indicating that agreement tends to be low when KL-divergence is high. Figure 2 illustrates this effect even more dramatically. Here we plot accuracy against token subsets of size $1000,2000, . ., N$ where tokens are added to the subsets according to their KL-divergence, selecting those with the highest values first. This demonstrates clearly that tokens with higher KLdivergence have lower inter-annotator agreement.

However, as discussed in sections 2.3 and 2.4, we decided on sentences as the preferred annotation level. Therefore, it is important to explore these relationships at the sentence level as well. Again, we start by looking at the Pearson correlation coefficient between f-score inter-annotator agreement (as described in section 2.1) and our active learning selection metrics:

\begin{tabular}{lrrr}
\hline & Ave KL & Max KL & 1-F \\
\hline \hline All Tokens & -0.090 & -0.145 & -0.143 \\
O Removed & -0.042 & -0.092 & -0.101 \\
\hline
\end{tabular}

Here $O$ Removed means that sentences are removed for which the annotators agree that there are no entity phrases (i.e. all tokens are labelled as being outside an entity phrase). This shows a relation-

ment a numeric representation by assigning 1 to tokens on which the annotators agree and 0 to tokens on which they disagree. 


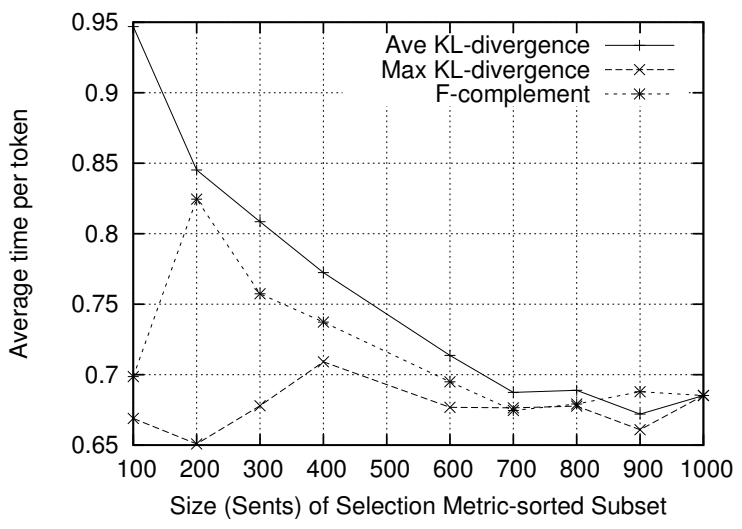

Figure 4: Annotation time plotted against selection metric-sorted document subsets.

ship very similar to what we observed at the token level: a negative correlation indicating that agreement is low when KL-divergence is high. Again, the effect of selecting informative examples is better illustrated with a plot. Figure 3 plots f-score agreement against sentence subsets sorted by our sentence level selection metrics. Lower agreement at the left of these plots indicates that the more informative examples according to our selection metrics are more difficult to annotate.

So, active learning makes the annotation more difficult. But, this raises a further question: What effect do more difficult examples have on annotation time? To investigate this, we once again start by looking at the Pearson correlation coefficient, this time between the annotation time and our selection metrics. However, as our sentence-level selection metrics affect the length of sentences selected, we normalise sentence-level annotation times by sentence length:

\begin{tabular}{lrrr}
\hline & Ave KL & Max KL & $1-\mathrm{F}$ \\
\hline \hline All Tokens & 0.157 & -0.009 & 0.082 \\
O Removed & 0.216 & -0.007 & 0.106 \\
\hline
\end{tabular}

Here we see a small positive correlations for averaged KL-divergence and f-complement indicating that sentences that score higher according to our selection metrics do generally take longer to annotate. Again, we can visualise this effect better by plotting average time against KL-sorted subsets (Figure 4). This demonstrates that sentences preferred by our selection metrics generally take longer to annotate.

\section{Conclusions and Future Work}

We have presented active learning experiments in a novel NER domain and investigated negative side effects. We investigated the relationship between informativity of an example, as determined by selective sampling metrics, and inter-annotator agreement. This effect has been quantified using the Pearson correlation coefficient and visualised using plots that illustrate the difficulty and time-intensiveness of examples chosen first by selective sampling. These measurements clearly demonstrate that selectively sampled examples are in fact more difficult to annotate. And, while sentence length and entities per sentence are somewhat confounding factors, we have also shown that selective sampling of informative examples appears to increase the time spent on individual examples.

High quality annotation is important for building accurate models and for reusability. While annotation quality suffers for selectively sampled examples, selective sampling nevertheless provided a dramatic cost reduction of $38.5 \%$ in a real annotation experiment, demonstrating the utility of active learning for bootstrapping NER in a new domain.

In future work, we will perform further investigations of the cost of resolving annotations for selectively sampled examples. And, in related work, we will use timing information to assess token, entity and sentence cost metrics for annotation. This should also lead to a better understanding of the relationship between timing information and sentence length for different selection metrics.

\section{Acknowledgements}

The work reported here, including the related development of the astronomy bootstrapping corpus and the active learning tools, were supported by Edinburgh-Stanford Link Grant (R36759) as part of the SEER project. We are very grateful for the time and resources invested in corpus preparation by our collaborators in the Institute for Astronomy, University of Edinburgh: Rachel Dowsett, Olivia Johnson and Bob Mann. We are also grateful to Melissa Kronenthal and Jean Carletta for help collecting data. 


\section{References}

Shlomo Argamon-Engelson and Ido Dagan. 1999. Committee-based sample selection for probabilistic classifiers. Journal of Artificial Intelligence Research, 11:335-360.

Jason Baldridge and Miles Osborne. 2004. Ensemblebased active learning for parse selection. In Proceedings of the 5th Conference of the North American Chapter of the Association for Computational Linguistics.

Markus Becker, Ben Hachey, Beatrice Alex, and Claire Grover. 2005. Optimising selective sampling for bootstrapping named entity recognition. In ICML-2005 Workshop on Learning with Multiple Views.

Thorsten Brants. 2000. Inter-annotator agreement for a German newspaper corpus. In Proceedings of the 2 nd International Conference on Language Resources and Evaluation (LREC-2000).

Jean Carletta, Amy Isard, Stephen Isard, Jacqueline C. Kowtko, Gwyneth Doherty-Sneddon, and Anne H. Anderson. 1997. The reliability of a dialogue structure coding scheme. Computational Linguistics, 23(1):13-31.

David. A. Cohn, Zoubin. Ghahramani, and Michael. I. Jordan. 1995. Active learning with statistical models. In G. Tesauro, D. Touretzky, and T. Leen, editors, Advances in Neural Information Processing Systems, volume 7, pages 705-712. The MIT Press.

Jenny Finkel, Shipra Dingare, Christopher Manning, Beatrice Alex Malvina Nissim, and Claire Grover. 2005. Exploring the boundaries: Gene and protein identification in biomedical text. BMC Bioinformatics. In press.

Rosie Jones, Rayid Ghani, Tom Mitchell, and Ellen Riloff. 2003. Active learning with multiple view feature sets. In ECML 2003 Workshop on Adaptive Text Extraction and Mining.

Jin-Dong Kim, Tomoko Ohta, Yoshimasa Tsuruoka, Yuka Tateisi, and Nigel Collier. 2004. Introduction to the bio-entity recognition task at JNLPBA. In Proceedings of the COLING 2004 International Joint Workshop on Natural Language Processing in Biomedicine and its Applications.

Dan Klein, Joseph Smarr, Huy Nguyen, and Christopher D. Manning. 2003. Named entity recognition with character-level models. In Proceedings the Seventh Conference on Natural Language Learning.

Mitchell P. Marcus, Beatrice Santorini, and Mary Ann Marcinkiewicz. 1994. Building a large annotated corpus of English: The Penn treebank. Computational Linguistics, 19(2):313-330.
Andrew McCallum and Kamal Nigam. 1998. Employing EM and pool-based active learning for text classification. In Proceedings of the 15th International Conference on Machine Learning.

Grace Ngai and David Yarowsky. 2000. Rule writing or annotation: Cost-efficient resource usage for base noun phrase chunking. In Proceedings of the 38th Annual Meeting of the Association for Computational Linguistics.

Patricia Robinson, Erica Brown, John Burger, Nancy Chinchor, Aaron Douthat, Lisa Ferro, and Lynette Hirschman. 1999. Overview: Information extraction from broadcast news. In Proceedings DARPA Broadcast News Workshop.

Erik F. Tjong Kim Sang. 2002. Introduction to the CoNLL-2002 shared task: Language-independent named entity recognition. In Proceedings of the 2002 Conference on Computational Natural Language Learning.

H. Sebastian Seung, Manfred Opper, and Haim Sompolinsky. 1992. Query by committee. In Computational Learning Theory.

Stephanie Strassel, Alexis Mitchell, and Shudong Huang. 2003. Multilingual resources for entity extraction. In Proceedings of the ACL 2003 Workshop on Multilingual and Mixed-language Named Entity Recognition.

Cynthia A. Thompson, Mary Elaine Califf, and Raymond J. Mooney. 1999. Active learning for natural language parsing and information extraction. In Proceedings of the 16th International Conference on Machine Learning. 